\title{
Chaos synchronization of identical Sprott systems by active control
}

\author{
Marcin Daszkiewicz \\ Institute of Theoretical Physics \\ University of Wroclaw pl. Maxa Borna 9, 50 - 206 Wroclaw, Poland \\ e - mail : marcin@ift.uni.wroc.pl
}

\begin{abstract}
In this article we synchronize by active control method all 19 identical Sprott systems provided in paper [10]. Particularly, we find the corresponding active controllers as well as we perform (as an example) the numerical synchronization of two Sprott-A models.
\end{abstract}




\section{Introduction}

In the last four decades there appeared a lot of papers dealing with so-called chaotic models, i.e., with such models whose dynamics is described by strongly sensitive with respect initial conditions, nonlinear differential equations. The most popular of them are: Lorenz system [1], Roessler system [2], Rayleigh-Benard system [3], Henon-Heiles system [4], jerk equation [5], Duffing equation [6], Lotka-Volter system [7], Liu system [8], Chen system [9] and Sprott system [10]. A lot of them have been applied in various fields of industrial and scientific divisions, such as, for example: Physics, Chemistry, Biology, Microbiology, Economics, Electronics, Engineering, Computer Science, Secure Communications, Image Processing and Robotics.

One of the most important problem of the chaos theory concerns so-called chaos synchronization phenomena. Since Pecora and Caroll [11] introduced a method to synchronize two identical chaotic systems, the chaos synchronization has received increasing attention due to great potential applications in many scientific discipline. Generally, there are known several methods of chaos synchronization, such as: OGY method [12, active control method [13]-[16], adaptive control method [17]-[21], backstepping method [22], [23], sampled-data feedback synchronization method [24], time-delay feedback method [25] and sliding mode control method [26]-[29].

In this article we synchronize by active control scheme all 19 identical Sprott systems provided in publication [10. Particularly, we establish the proper so-called active controllers with use of the Lyapunov stabilization theory [30]. It should be noted, however, that some types of Sprott model have been already synchronized with use of the active control method in papers [31] and 32]. Apart of that there has been synchronized the Sprott systems in the framework of adaptive control scheme in articles [33] and [34].

The paper is organized as follows. In second Section we recall the main result of Sprott article [10], i.e., we provide Table 1 including dynamics of all 19 Sprott chaotic systems.

In Section 3 we remaind the basic concepts of active synchronization method, while in Section 4 we consider as an example the synchronization of two identical Sprott-A models. The fifth Section is devoted to the main result of the paper, and it provides in Table 2 the active controllers which synchronize all identical Sprott systems. The conclusions and final remarks are discussed in the last Section.

\section{Sprott systems}

In this Section we recall the main result of paper [10], in which there has been performed a systematic examination of general three-dimensional ordinary differential equations with quadratic nonlinearities. Particularly, it has been uncovered 19 distinct simple examples of chaotic flows (so-called Sprott systems) listed in Table 1. 


\begin{tabular}{cccc}
\hline type & 1st equation & 2nd equation & 3rd equation \\
\hline $\mathrm{A}$ & $\dot{x}_{1}=x_{2}$ & $\dot{x}_{2}=-x_{1}+x_{2} x_{3}$ & $\dot{x}_{3}=1-x_{2}^{2}$ \\
$\mathrm{~B}$ & $\dot{x}_{1}=x_{2} x_{3}$ & $\dot{x}_{2}=x_{1}-x_{2}$ & $\dot{x}_{3}=1-x_{1} x_{2}$ \\
$\mathrm{C}$ & $\dot{x}_{1}=x_{2} x_{3}$ & $\dot{x}_{2}=x_{1}-x_{2}$ & $\dot{x}_{3}=1-x_{1}^{2}$ \\
$\mathrm{D}$ & $\dot{x}_{1}=-x_{2}$ & $\dot{x}_{2}=x_{1}+x_{3}$ & $\dot{x}_{3}=x_{1} x_{3}+3 x_{2}^{2}$ \\
$\mathrm{E}$ & $\dot{x}_{1}=x_{2} x_{3}$ & $\dot{x}_{2}=x_{1}^{2}-x_{2}$ & $\dot{x}_{3}=1-4 x_{1}$ \\
$\mathrm{~F}$ & $\dot{x}_{1}=x_{2}+x_{3}$ & $\dot{x}_{2}=-x_{1}+0.5 x_{2}$ & $\dot{x}_{3}=x_{1}^{2}-x_{3}$ \\
$\mathrm{G}$ & $\dot{x}_{1}=0.4 x_{1}+x_{3}$ & $\dot{x}_{2}=x_{1} x_{3}-x_{2}$ & $\dot{x}_{3}=-x_{1}+x_{2}$ \\
$\mathrm{H}$ & $\dot{x}_{1}=-x_{2}+x_{3}^{2}$ & $\dot{x}_{2}=x_{1}+0.5 x_{2}$ & $\dot{x}_{3}=x_{1}-x_{3}$ \\
$\mathrm{I}$ & $\dot{x}_{1}=-0.2 x_{2}$ & $\dot{x}_{2}=x_{1}+x_{3}$ & $\dot{x}_{3}=x_{1}+x_{2}^{2}-x_{3}$ \\
$\mathrm{~J}$ & $\dot{x}_{1}=2 x_{3}$ & $\dot{x}_{2}=-2 x_{2}+x_{3}$ & $\dot{x}_{3}=-x_{1}+x_{2}+x_{2}^{2}$ \\
$\mathrm{~K}$ & $\dot{x}_{1}=x_{1} x_{2}-x_{3}$ & $\dot{x}_{2}=x_{1}-x_{2}$ & $\dot{x}_{3}=x_{1}+0.3 x_{3}$ \\
$\mathrm{~L}$ & $\dot{x}_{1}=x_{2}+3.9 x_{3}$ & $\dot{x}_{2}=0.9 x_{1}^{2}-x_{2}$ & $\dot{x}_{3}=1-x_{1}$ \\
$\mathrm{M}$ & $\dot{x}_{1}=-x_{3}$ & $\dot{x}_{2}=-x_{1}^{2}-x_{2}$ & $\dot{x}_{3}=1.7+1.7 x_{1}+x_{2}$ \\
$\mathrm{~N}$ & $\dot{x}_{1}=-2 x_{2}$ & $\dot{x}_{2}=x_{1}+x_{3}^{2}$ & $\dot{x}_{3}=1+x_{2}-2 x_{1}$ \\
$\mathrm{O}$ & $\dot{x}_{1}=x_{2}$ & $\dot{x}_{2}=x_{1}-x_{3}$ & $\dot{x}_{3}=x_{1}+x_{1} x_{3}+2.7 x_{2}$ \\
$\mathrm{P}$ & $\dot{x}_{1}=2.7 x_{2}+x_{3}$ & $\dot{x}_{2}=-x_{1}+x_{2}^{2}$ & $\dot{x}_{3}=x_{1}+x_{2}$ \\
$\mathrm{Q}$ & $\dot{x}_{1}=-x_{3}$ & $\dot{x}_{2}=x_{1}-x_{2}$ & $\dot{x}_{3}=3.1 x_{1}+x_{2}^{2}+0.5 x_{3}$ \\
$\mathrm{R}$ & $\dot{x}_{1}=0.9-x_{2}$ & $\dot{x}_{2}=0.4+x_{3}$ & $\dot{x}_{3}=x_{1} x_{2}-x_{3}$ \\
$\mathrm{~S}$ & $\dot{x}_{1}=-x_{1}-4 x_{2}$ & $\dot{x}_{2}=x_{1}+x_{3}^{2}$ & $\dot{x}_{3}=1+x_{1}$ \\
\hline
\end{tabular}

Table 1. The Sprott systems.

\section{Chaos synchronization by active control - general prescription}

In this Section we remaind the general scheme of chaos synchronization of two systems by so-called active control procedure [13]-[16]. Let us start with the following master mode $]^{1}$

$$
\dot{x}=A x+F(x),
$$

where $x=\left[x_{1}, x_{2}, \ldots, x_{n}\right]$ is the state of the system, A denotes the $n \times n$ matrix of the system parameters and $F(x)$ plays the role of the nonlinear part of the differential equation (1). The slave model dynamics is described by

$$
\dot{y}=B y+G(y)+u,
$$

with $y=\left[y_{1}, y_{2}, \ldots, y_{n}\right]$ being the state of the system, $B$ denoting the $n$-dimensional quadratic matrix of the system, $G(y)$ playing the role of nonlinearity of the equation (2) and $u=\left[u_{1}, u_{2}, \ldots, u_{n}\right]$ being the active controller of the slave model. Besides, it should be mentioned that for matrices $A=B$ and functions $F=G$ the states $x$ and $y$ describe

$$
1 \frac{d o}{d t}=\dot{o}
$$


two identical chaotic systems. In the case $A \neq B$ or $F \neq G$ they correspond to the two different chaotic models.

Let us now provide the following synchronization error vector

$$
e=y-x
$$

which in accordance with (1) and (2) obeys

$$
\dot{e}=B y-A x+G(y)-F(x)+u \text {. }
$$

In active control method we try to find such a controller $u$, which synchronizes the state of the master system (1) with the state of the slave system (2) for any initial condition

$x_{0}=x(0)$ and $y_{0}=y(0)$. In other words we design a controller $u$ in such a way that for system (4) we have

$$
\lim _{t \rightarrow \infty}\|e(t)\|=0
$$

for all initial conditions $e_{0}=e(0)$. In order to establish the synchronization (4) we use the Lyapunov stabilization theory [30]. It means, that if we take as a candidate Lyapunov function of the form

$$
V(e)=e^{T} P V(e) e,
$$

with $P$ being a positive $n \times n$ matrix, then we wish to find the active controller $u$ so that

$$
\dot{V}(e)=-e^{T} Q V(e) e,
$$

where $\mathrm{Q}$ is a positive definite $n \times n$ matrix as well. Then the systems (1) and (2) remain synchronized.

\section{The example: chaos synchronization of (identical) Sprott-A systems}

In accordance with two pervious Sections the master Sprott-A system is described by the following dynamics (see Table 1)

$$
\left\{\begin{array}{l}
\dot{x}_{1}=x_{2} \\
\dot{x}_{2}=-x_{1}+x_{2} x_{3} \\
\dot{x}_{3}=1-x_{2}^{2},
\end{array}\right.
$$


where functions $x_{1}, x_{2}$ and $x_{3}$ denote the states of the system; its slave Sprott-A partner is given by

$$
\left\{\begin{array}{l}
\dot{y}_{1}=y_{2}+u_{1} \\
\dot{y}_{2}=-y_{1}+y_{2} y_{3}+u_{2} \\
\dot{y}_{3}=1-y_{2}^{2}+u_{3},
\end{array}\right.
$$

with active controllers $u_{1}, u_{2}$ and $u_{3}$ respectively. Using (8) and (9) one can check that the dynamics of synchronization errors $e_{i}=y_{i}-x_{i}$ is obtained as $\mathrm{s}^{2}$

$$
\left\{\begin{array}{l}
\dot{e}_{1}=e_{2}+u_{1} \\
\dot{e}_{2}=-e_{1}+y_{2} y_{3}-x_{2} x_{3}+u_{2} \\
\dot{e}_{3}=-e_{2}\left(y_{2}+x_{2}\right)+u_{3} .
\end{array}\right.
$$

Besides, if we define the positive Lyapunov function by ${ }^{3}$

$$
V(e)=\frac{1}{2}\left(e_{1}^{2}+e_{2}^{2}+e_{3}^{2}\right)
$$

then for the following choice of control functions

$$
\left\{\begin{array}{l}
u_{1}=-\left(e_{1}+e_{2}\right) \\
u_{2}=e_{1}-e_{2}-y_{2} y_{3}+x_{2} x_{3} \\
u_{3}=e_{2}\left(y_{2}+x_{2}\right)-e_{3},
\end{array}\right.
$$

we have 4

$$
\dot{V}(e)=-\left(e_{1}^{2}+e_{2}^{2}+e_{3}^{2}\right)
$$

Such a result means (see general prescription) that the identical Sprott-A systems (8) and (9) are synchronized for all initial conditions with active controllers (12).

Let us now illustrate the above considerations by the proper numerical calculations.

First of all, we solve the Sprott-A system with two different sets of initial conditions

$$
\left(x_{01}, x_{02}, x_{03}\right)=(1,0.05,0.05) \text {, }
$$

\footnotetext{
${ }^{2}$ See also formula $\sqrt{4}$.

${ }^{3}$ The matrix $P=1$ in the formula $(6)$.

${ }^{4}$ The matrix $Q=1$ in the formula $(7)$.
} 
and

$$
\left(y_{01}, y_{02}, y_{03}\right)=(1.05,0.15,0)
$$

respectively. The results are presented on Figure $\mathbf{1}$ - one can see that there exist (in fact) the divergences between both trajectories. Next, we find the solutions for the master system (8) (the $x$-trajectory) and for its slave partner (9) with active controllers (12) (the $y$-trajectory) for initial data (14) and (15) respectively. Now, we see that the corresponding trajectories become synchronized - the vanishing in time error functions $e_{i}=y_{i}-x_{i}$ are presented on Figure 2. Additionally, we repeat the above numerical procedure for two another sets of initial data: $x_{0}=(0,0.15,0.05)$ and $y_{0}=(0.05,0.05,0)$; the obtained results are presented on Figures 3 and 4 respectively.

\section{Chaos synchronization of identical Sprott systems}

The used in pervious Section algorithm can be applied to the case of all remaining Sprott systems as well. The obtained results are summarized in Table 2, i.e., there are listed controllers $u_{1}, u_{2}$ and $u_{3}$ for which the proper identical Sprott systems become synchronized for arbitrary initial conditions $x_{01}, x_{02}$ and $x_{03}$ as well as $y_{01}, y_{02}$ and $y_{03}$. However, as it was already mentioned in Introduction, the control functions for Sprott-L and Sprott-M models have been provided in paper [31].

\begin{tabular}{cccc}
\hline type & 1st controller & 2nd controller & 3rd controller \\
\hline $\mathrm{A}$ & $u_{1}=-\left(e_{1}+e_{2}\right)$ & $u_{2}=e_{1}-e_{2}-y_{2} y_{3}+$ & $u_{3}=e_{2}\left(y_{2}+x_{2}\right)-e_{3}$ \\
& $u_{1}=x_{2} x_{3}-y_{2} y_{3}-e_{1}$ & $+x_{2} x_{3}$ & \\
$\mathrm{~B}$ & $u_{2}=-e_{1}$ & $u_{3}=y_{1} y_{2}-x_{1} x_{2}-e_{3}$ \\
$\mathrm{C}$ & $u_{1}=x_{2} x_{3}-y_{2} y_{3}-e_{1}$ & $u_{2}=-e_{1}$ & $u_{3}=\left(x_{1}+y_{1}\right) e_{1}-e_{3}$ \\
$\mathrm{D}$ & $u_{1}=e_{2}-e_{1}$ & $u_{2}=-\left(e_{1}+e_{2}+e_{3}\right)$ & $u_{3}=x_{1} x_{3}-3\left(y_{2}+x_{2}\right)$. \\
& $u_{1}=x_{1} x_{3}-y_{1} y_{3}-e_{1}$ & $u_{2}=-\left(y_{1}+x_{1}\right) e_{1}$ & $\cdot e_{2}-e_{3}-y_{1} y_{2}$ \\
$\mathrm{E}$ & $u_{1}=-\left(e_{1}+e_{2}+e_{3}\right)$ & $u_{2}=e_{1}-1.5 e_{2}$ & $u_{3}=4 e_{1}-e_{2}$ \\
$\mathrm{~F}$ & $u_{1}=-\left(1.4 e_{1}+e_{3}\right)$ & $u_{2}=x_{1} x_{3}-y_{1} y_{3}$ & $u_{3}=e_{1}-\left(e_{2}+e_{3}\right)$ \\
$\mathrm{G}$ & $u_{1}=e_{2}-\left(y_{3}+x_{3}\right)$. & $u_{2}=-\left(e_{1}+1.5 e_{2}\right)$ & $u_{3}=-e_{1}$ \\
$\mathrm{H}$ & $\cdot e_{3}-e_{1}$ & $u_{2}=-\left(e_{1}+e_{2}+e_{3}\right)$ & $u_{3}=-\left(e_{1}+\left(y_{2}+x_{2}\right)\right.$. \\
& $u_{1}=0.2 e_{2}-e_{1}$ & & $\left.\cdot e_{2}\right)$ \\
$\mathrm{I}$ & $u_{1}=-\left(e_{1}+2 e_{3}\right)$ & $u_{2}=e_{2}-e_{3}$ & $u_{3}=e_{1}-\left(1+y_{2}+x_{3}\right)$. \\
& $u_{1}=e_{3}-e_{1}-y_{1} y_{2}+$ & $u_{2}=-e_{1}$ & $\left.\cdot e_{2}-e_{3}\right)$ \\
$\mathrm{J}$ & $x_{1} x_{2}$ & $u_{3}=-\left(e_{1}+1.3 e_{3}\right)$ \\
$\mathrm{K}$ & $u_{1}=-\left(e_{1}+e_{2}+3.9 e_{3}\right)$ & $u_{2}=-0.9\left(y_{1}+x_{1}\right) e_{1}$ & $u_{3}=e_{1}-e_{3}$
\end{tabular}




$\begin{array}{cccc}\mathrm{M} & u_{1}=e_{3}-e_{1} & u_{2}=-e_{1}\left(y_{1}+x_{1}\right) & u_{3}=-\left(1.7 e_{1}+e_{2}+e_{3}\right) \\ \mathrm{N} & u_{1}=2 e_{2}-e_{1} & u_{2}=-\left(e_{1}+\left(y_{3}+x_{3}\right) .\right. & u_{3}=2 e_{1}-e_{2}-e_{3} \\ & & \left.\cdot e_{3}+e_{2}\right) & \\ \mathrm{O} & u_{1}=-\left(e_{1}+e_{2}\right) & u_{2}=-\left(e_{1}+e_{2}\right)+e_{3} & u_{3}=-\left(e_{1}+2.7 e_{2}+\right. \\ & & & \left.+e_{3}\right)+x_{1} x_{2}-y_{1} y_{2} \\ \mathrm{P} & u_{1}=-\left(e_{1}+2.7 e_{2}+\right. & u_{2}=e_{1}-\left(y_{2}+x_{2}\right) . & u_{3}=-\left(e_{1}+e_{2}+e_{3}\right) \\ & \left.+e_{3}\right) & \left.\cdot e_{2}-e_{2}\right) & \\ \mathrm{Q} & u_{2}=e_{1} & u_{3}=-\left(3.1 e_{1}+\left(y_{2}+x_{2}\right) .\right. \\ & u_{1}=e_{3}-e_{1} & \left.e_{2}\right)-1.5 e_{3} \\ \mathrm{R} & u_{1}=-e_{1}+e_{2} & u_{2}=-\left(e_{2}+e_{3}\right) & u_{3}=x_{1} x_{2}-y_{1} y_{2} \\ \mathrm{~S} & u_{1}=4 e_{2} & u_{2}=-\left(e_{1}+\left(y_{3}+x_{3}\right) .\right. & u_{3}=-\left(e_{1}+e_{3}\right) \\ & & \left.\cdot e_{3}\right)-e_{2} & \\ & & & \end{array}$

Table 2. The active controllers for Sprott systems.

\section{Final remarks}

In this article we synchronize all identical Sprott systems defined in paper [10] with use of the active control method. Particularly, we find the corresponding so-called active controllers listed in Table 2. As an example we also study numerically synchronization of Sprott-A model defined by the formulas (8) and (9).

It should be noted that the presented investigations can be extended in various ways. For example, one may consider synchronization of Sprott models with use of others mentioned in Introduction methods. The works in this direction already started and are in progress.

\section{References}

[1] E.N. Lorenz, J. Atmos. Sci. 20, 130 (1963)

[2] O.E. Roessler, Phys. Lett. A 57, 397 (1976)

[3] A.V. Getling, "Rayleigh-Benard Convection: Structures and Dynamics", World Scientific, 1998

[4] M. Henon, C. Heiles, AJ. 69, 73 (1964)

[5] J.C. Sprott, Am J Phys. 65, 537 (1997)

[6] G. Duffinng, "Erzwungene Schwingungen bei Vernderlicher Eigenfrequenz", F. Vieweg u. Sohn, Braunschweig, 1918. 
[7] V. Volterra "Variations and fluctuations of the number of indviduals in animal species living together. In Animal Ecology" McGraw-Hill, 1931. Translated from 1928 edition by R. N. Chapman.

[8] C. Liu, T. Liu, L. Liu, K. Liu, Chaos, Solitons and Fractals 22, 1031 (2004)

[9] G. Chen, T. Ueta, Journal of Bifurcation and Chaos 9, 1465 (1999)

[10] J.C. Sprott, Phys. Rev. E 50, 647 (1994)

[11] L.M. Pecora, T.L. Carroll, Phys. Rev. Lett 64, 821 (1990)

[12] E. Ott, C. Grebogi, J.A. Yorke, Phys. Rev. Lett 64, 1196 (1990)

[13] M.C. Ho, Y.C. Hung, Phys. Lett. A 301, 424 (2002)

[14] H.K. Chen, Chaos, Solitons and Fractals 23, 1245 (2005)

[15] V. Sundarapandian, Int. Journ. of Comp. Sc. and Eng. 3, 2145 (2011)

[16] V. Sundarapandian, Int. Journ. of Appl. Sc. and Tech. 3, 1 (2011)

[17] T.L. Liao, S.H. Tsai, Chaos, Solitons and Fractals 11, 1387 (2000)

[18] V. Sundarapandian, Int. Journ. of Cont. Theory and Comp. Model. 1, 1 (2011)

[19] V. Sundarapandian, Int. Journ. of Cont. Theory and Comp. Model. 1, 1 (2011)

[20] V. Sundarapandian, Int. Journ. of Comp. Eng. and Applications 1, 127 (2011)

[21] V. Sundarapandian, R. Karthikeyan, Int. Journ. of Inf. Tech. and Comp. Sc. 1, 49 (2011)

[22] Y.G. Yu, S.C. Zhang, Chaos, Solitons and Fractals, 27, 1369 (2006)

[23] X. Wu, J. Lu, Chaos, Solitons and Fractals, 18, 721 (2003)

[24] T. Yang, L.O. Chua, Int. J Bifurcat. Chaos 9, 215 (1999)

[25] J.H. Park, O.M. Kwon, Chaos, Solitons and Fractals 17, 709 (2003)

[26] V. Sundarapandian, Int. Journ. of Cont. Theory and Comp. Model. 1, 15 (2011)

[27] V. Sundarapandian, Int. Journ. of Comp. Sc. and Eng. 3, 2163 (2011)

[28] V. Sundarapandian, Int. Journ. on Inf. Sys. and Tech. 1, 20 (2011)

[29] V. Sundarapandian, S. Sivaperumal, Int. Journ. of Arch. Comp. 9, 274 (2012) 
[30] A.M. Lyapunov, "The General Problem of the Stability of Motion" (In Russian), Doctoral dissertation, Univ. Kharkov 1892. English translation: "Stability of Motion", Academic Press, New-York and London, 1966

[31] S. Vaidyanathan, Int. Journ. of Cont. Theory and Comp. Model. 2, 21 (2012)

[32] D. Xu, Adv. Theor. Appl. Mech. 3, 195 (2010)

[33] S. Vaidyanathan, Int. Journ. of Soft Comp. Math. and Cont. 1, 1 (2012)

[34] S. Vaidyanathan, Int. Journ. of Inf. and Comp. Sec. 1, 13 (2011) 


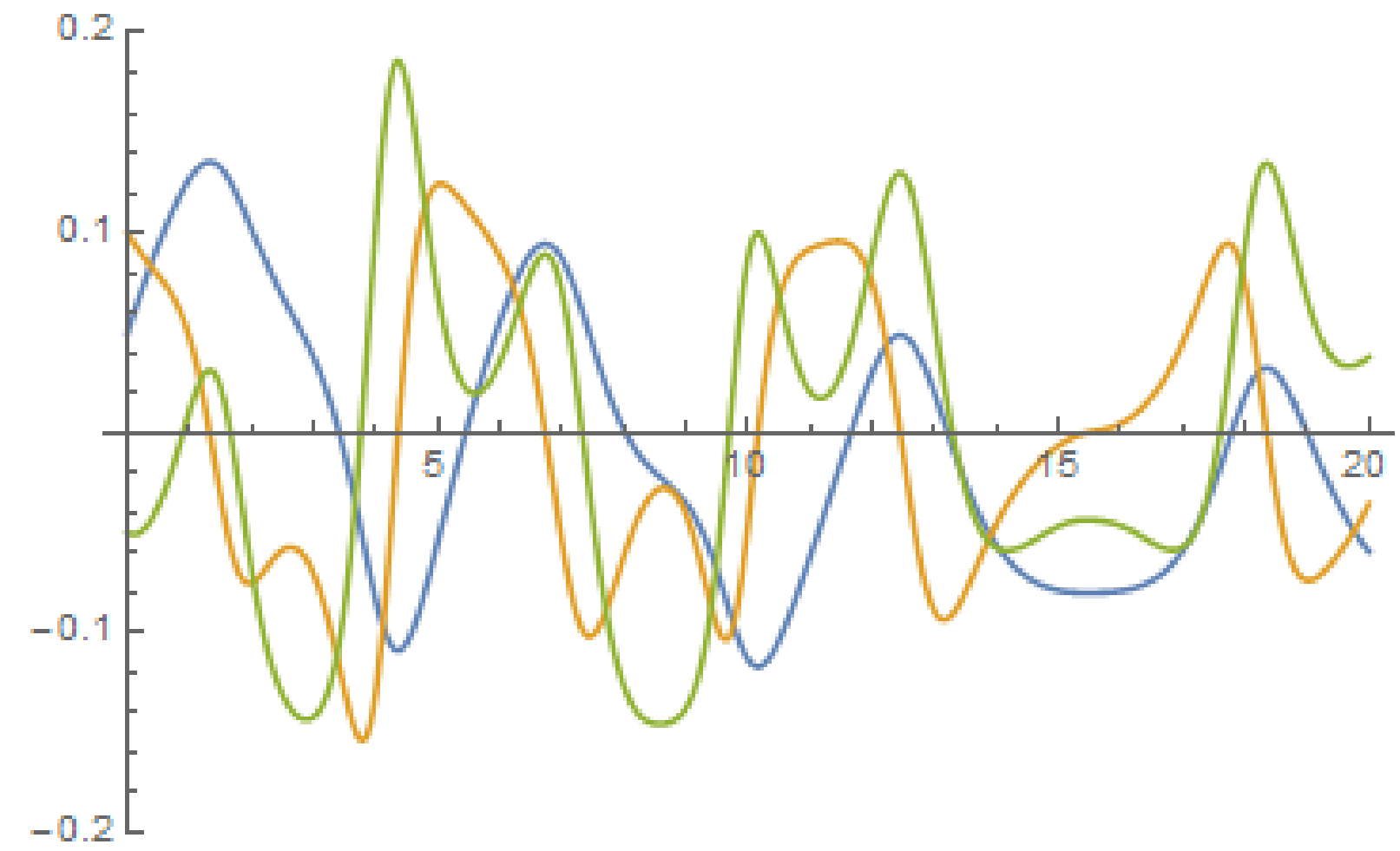

Figure 1: The error functions $e_{i}=y_{i}-x_{i}$ for Sprott-A model defined by the system (8) with the initial conditions (14) (the $x$-trajectory) and with the initial conditions (15) (the $y$-trajectory). The blue line corresponds to the $e_{1}$-error function, the orange one - to $e_{2}$ and the green one - to $e_{3}$ respectively. 


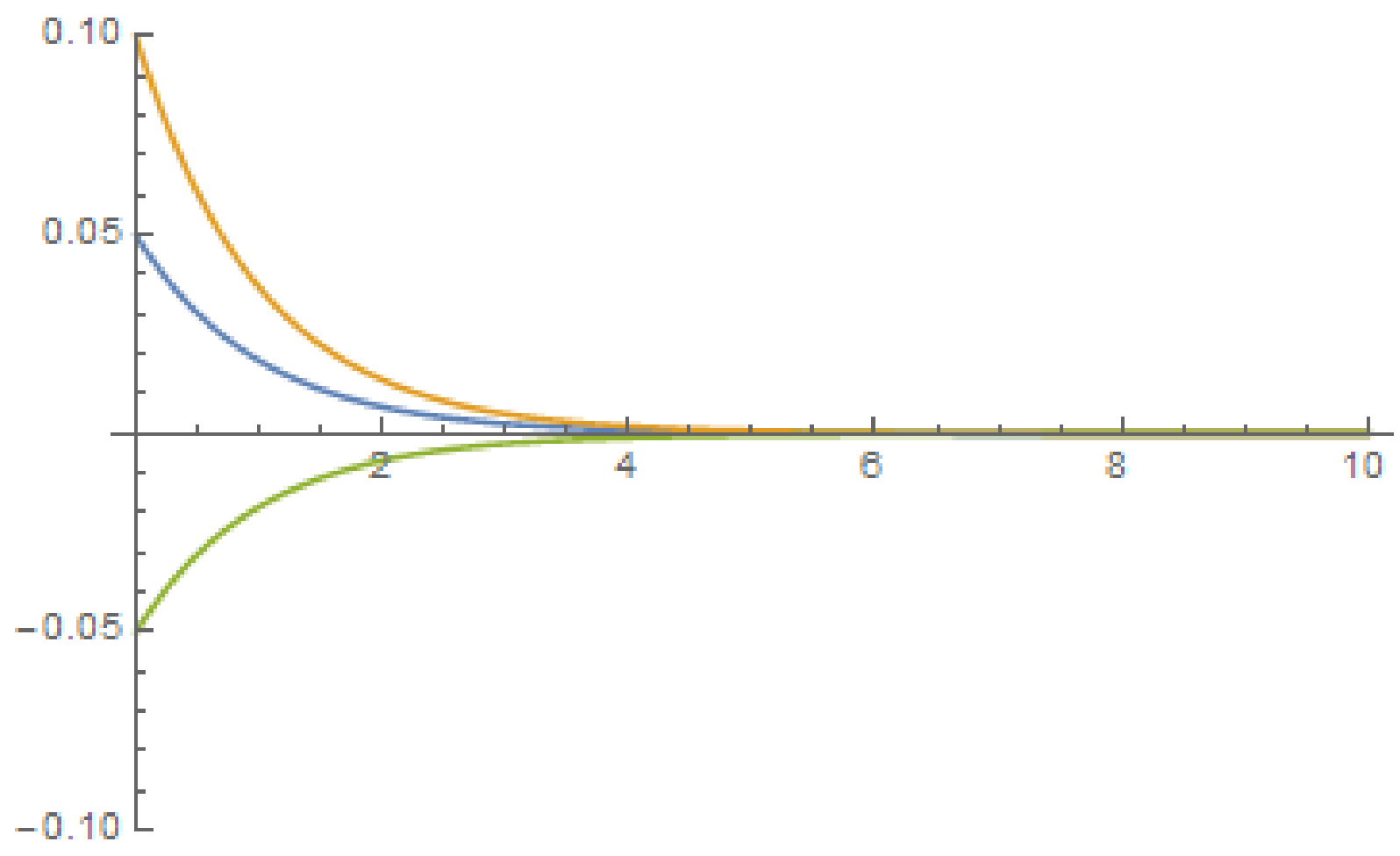

Figure 2: The error functions $e_{i}=y_{i}-x_{i}$ for synchronized Sprott-A model defined by the master system (8) with the initial conditions (14) (the $x$-trajectory) and by the slave system (9) with the initial conditions (15) (the $y$-trajectory). The blue line corresponds to the $e_{1}$-error function, the orange one - to $e_{2}$ and the green one - to $e_{3}$ respectively. 


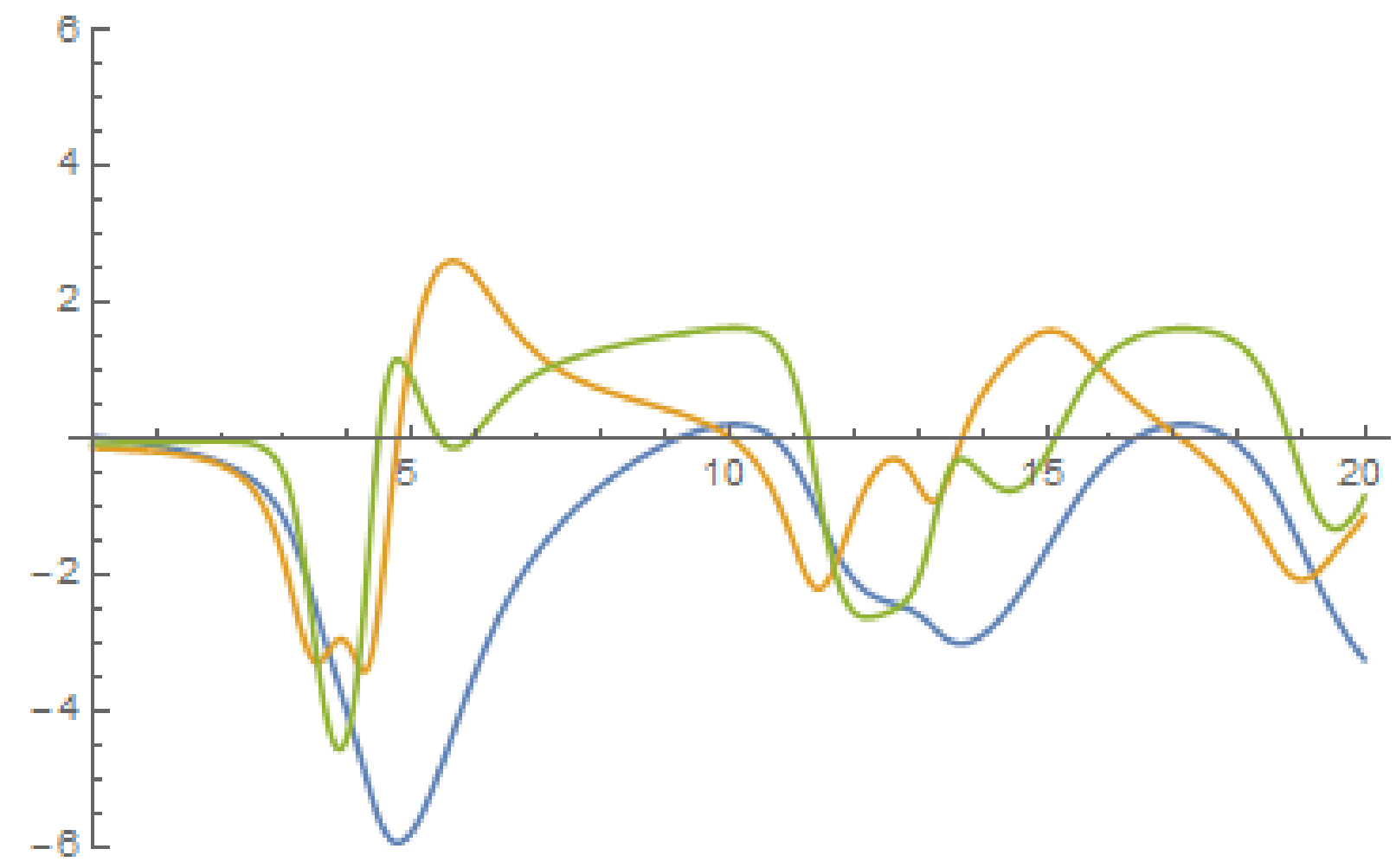

Figure 3: The error functions $e_{i}=y_{i}-x_{i}$ for Sprott-A model defined by the system (8) with the initial conditions $x_{0}=(0,0.15,0.05)$ (the $x$-trajectory) and with the initial conditions $y_{0}=(0.05,0.05,0)$ (the $y$-trajectory). The blue line corresponds to the $e_{1}$-error function, the orange one - to $e_{2}$ and the green one - to $e_{3}$ respectively. 


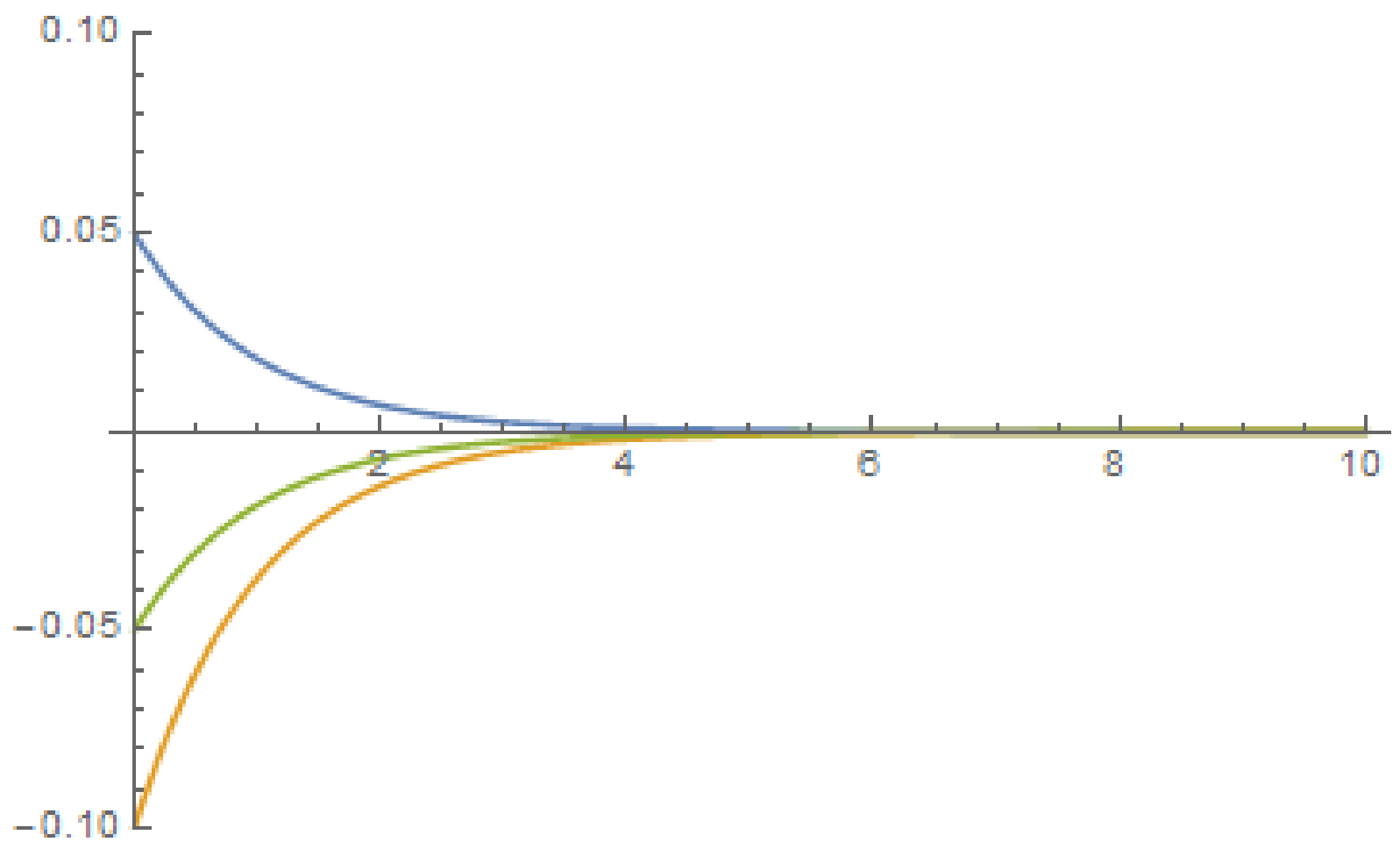

Figure 4: The error functions $e_{i}=y_{i}-x_{i}$ for synchronized Sprott-A model defined by the master system (8) with the initial conditions $x_{0}=(0,0.15,0.05)$ (the $x$-trajectory) and by the slave system (9) with the initial conditions $y_{0}=(0.05,0.05,0)$ (the $y$-trajectory). The blue line corresponds to the $e_{1}$-error function, the orange one - to $e_{2}$ and the green one - to $e_{3}$ respectively. 